\title{
Role of JNK and NF-kB pathways in Porphyromonas gingivalis LPS-induced vascular cell adhesion molecule-1 expression in human aortic endothelial cells
}

\author{
BIN LIU ${ }^{1,3}$, JIA WANG ${ }^{1,3}$, LAN CHENG $^{2,3}$ and JINGPING LIANG ${ }^{1,3}$ \\ Departments of ${ }^{1}$ Endodontics and Operative Dentistry and ${ }^{2}$ Periodontology, Ninth People's Hospital, School of Medicine, \\ Shanghai Jiao Tong University, Shanghai 200011; ${ }^{3}$ Shanghai Key Laboratory of Stomatology, Shanghai 200011, P.R. China
}

Received May 2, 2013; Accepted September 5, 2013

DOI: $10.3892 / \mathrm{mmr} .2013 .1685$

\begin{abstract}
An increasing number of studies have shown a correlation between Porphyromonas gingivalis ( $P$. gingivalis) infection and atherosclerosis. A recent study demonstrated that the expression of vascular cell adhesion molecule-1 (VCAM-1) was induced by $P$. gingivalis lipopolysaccharide (LPS) in human aortic endothelial cells (HAECs). The activation of p38 mitogen-activated protein kinase (p38 MAPK) was at least partially involved in this process. Those results suggested the potential involvement of $P$. gingivalis LPS in the pathogenesis of atherosclerosis. However, the mechanism involved in $P$. gingivalis LPS-induced VCAM-1 production has not yet been elucidated. The present study examined the role of the c-Jun N-terminal kinase (JNK) and nuclear factor- $\kappa \mathrm{B}(\mathrm{NF}-\kappa \mathrm{B})$ cell signaling pathways in $P$. gingivalis LPS-induced VCAM-1 expression in HAECs. Western blotting was used to investigate the activation of JNK and NF- $\kappa \mathrm{B}$ pathways in HAECs exposed to $P$. gingivalis LPS. Following this, specific pharmacological inhibitors were introduced and the protein production of VCAM-1 was studied. The results showed that the JNK and NF- $\kappa \mathrm{B}$ pathways in HAECs were capable of being activated by $P$. gingivalis LPS. The inhibition of NF- $\kappa$ B by SN50 significantly attenuated $P$. gingivalis LPS-induced VCAM-1 expression, while the inhibition of JNK by SP600125 enhanced VCAM-1 expression in $P$. gingivalis LPS-treated HAECs. Therefore, the results indicated that $\mathrm{NF}-\kappa \mathrm{B}$ was essential for the $P$. gingivalis LPS-induced VCAM-1 expression in HAECs and that JNK may be a suppressor of VCAM-1 expression in HAECs.
\end{abstract}

Correspondence to: Professor Jingping Liang, Department of Endodontics and Operative Dentistry, Ninth People's Hospital, School of Medicine, Shanghai Jiao Tong University, 639 Zhi Zao Ju Road, Shanghai 200011, P.R. China

E-mail: liangjingping@yahoo.com

Key words: Porphyromonas gingivalis, endothelial cells, JNK mitogen-activated protein kinases, lipopolysaccharide, nuclear factor- $\kappa \mathrm{B}$, vascular cell adhesion molecule-1

\section{Introduction}

Periodontal disease, a chronic inflammatory disease of the periodontium, is initiated by bacterial infections, which may eventually result in the loss of teeth. Porphyromonas gingivalis (P. gingivalis), a Gram-negative and obligate anaerobic bacterium, has long been considered to be an important pathogen involved in human periodontal disease. Previous studies have suggested a correlation between periodontal disease and atherosclerosis and that $P$. gingivalis infection may accelerate the development of atherosclerosis (1-4).

Lipopolysaccharide (LPS) is the main component of the cell wall of Gram-negative bacteria (5) and has been increasingly suggested to be responsible for the activation of vascular endothelial cells (VECs) and the initiation of the atherosclerotic process $(6,7)$. P. gingivalis LPS, which is important in $P$. gingivalis infection (8), may be released into the bloodstream following scaling and root planing or even mastication in patients with severe periodontal disease, resulting in elevated levels of circulating endotoxin, which has been shown to be correlated with an increased risk of atherosclerotic incident (9-13). It has previously been shown that $P$. gingivalis LPS has a similar ability to that of Escherichia coli (E. coli) to activate human aortic endothelial cells (HAECs) by elevating the gene expression and protein production of vascular cell adhesion molecule-1 (VCAM-1) $(4,14)$. VCAM-1 belongs to the immunoglobulin superfamily, is important in leukocyte recruitment to sites of inflammation and accelerates the development of atherosclerosis (15-17). Therefore, $P$. gingivalis LPS-induced VCAM-1 expression may contribute to the initiation of the atherosclerotic process.

Multiple signaling mechanisms, in a number of cell lines, have been demonstrated to be involved in the induction of VCAM-1 expression in response to various stimuli. This suggests that there are divergent pathways leading to VCAM-1 expression, depending on the nature of the stimulus and the cell type $(4,18-21)$. A major mechanism through which signals from extracellular stimuli are transmitted to the nucleus involves the activation of intracellular kinases, including those belonging to the mitogen-activated protein kinase (MAPK) superfamily. p38 MAPK and c-Jun N-terminal kinase (JNK) are two distinct and parallel MAPK pathways that have been 
demonstrated to predominantly be involved in the regulation of cellular inflammation in response to stimuli, including the E. coli LPS-induced upregulation of VCAM-1 (22-26).

Previous studies have shown the activation of p38 MAPK and JNK by $P$. gingivalis LPS in a number of cell lines; however, studies concerning human VECs are limited (27-29). In addition, the majority of the previous studies investigated the response of human umbilical VECs (HUVECs) to $P$. gingivalis LPS and were, therefore, less relevant to atherosclerosis. We recently demonstrated the involvement of the p38 MAPK pathway in $P$. gingivalis LPS-induced VCAM-1 expression in HAECs (4). However, the mechanisms responsible for $P$. gingivalis LPS-induced VCAM-1 expression in VECs have yet to be elucidated. Therefore, further study is required to demonstrate whether the activation of JNK by $P$. gingivalis LPS is associated with VCAM-1 expression in HAECs. In addition, it is of interest that many of the genes regulated by MAPKs are dependent on nuclear factor $-\kappa \mathrm{B}(\mathrm{NF}-\kappa \mathrm{B})$ for transcription. $\mathrm{NF}-\kappa \mathrm{B}$ has also been shown to be involved in the expression of adhesion molecules at the transcriptional level, in various cell types $(19,20,30)$. Since it has been shown that discrepancies in chemical structures, immunobiological activities and the activation of intracellular signaling pathways exist between $P$. gingivalis and $E$. coli LPS $(31,32), E$. coli LPS was also introduced in the present study.

The experiments in the present study were performed to investigate the roles of $\mathrm{JNK}$ and $\mathrm{NF}-\kappa \mathrm{B}$ in $P$. gingivalis LPS-induced VCAM-1 protein expression in HAECs. It was demonstrated that $\mathrm{NF}-\kappa \mathrm{B}$ was required for this expression, whereas JNK was a suppressor of VCAM-1 expression. These results provide a novel insight into the mechanisms of $P$. gingivalis LPS action in the initiation of atherosclerosis.

\section{Materials and methods}

Reagents. $P$. gingivalis LPS was purchased from Invitrogen Life Technologies (San Diego, CA, USA), while E. coli LPS (055:B5) and $\beta$-actin monoclonal antibody were obtained from Sigma-Aldrich (St. Louis, MO, USA). The monoclonal antibodies for JNK (56G8), phosphorylated JNK (p-JNK; G9), NF- $\kappa$, and nuclear factor of $\kappa$ light polypeptide gene enhancer in B-cells inhibitor- $\alpha(\mathrm{I} \kappa \mathrm{B}-\alpha)$ were obtained from Cell Signaling Technology, Inc. (Danvers, MA, USA). VCAM-1 (E-10) monoclonal antibody was obtained from Santa Cruz Biotechnology, Inc. (Santa Cruz, CA, USA). The JNK (JNK Inhibitor II, SP600125) and NF- אB (SN50) inhibitors were obtained from Calbiochem (San Diego, CA, USA). SP600125 was dissolved in dimethylsulfoxide (DMSO), while SN50 was dissolved in ultrapure water at a concentration of $50 \mathrm{mM}$ for stock solutions.

Cells and culture conditions. HAECs and Endothelial Cell Medium (ECM) were obtained from ScienCell (Carlsbad, CA, USA). ECM was prepared for use by mixing $5 \mathrm{ml}$ Endothelial Cell Growth Supplement (ECGS), $5 \mathrm{ml}$ penicillin/ streptomycin solution (P/S) and $25 \mathrm{ml}$ fetal bovine serum (FBS) into $500 \mathrm{ml}$ basal medium, in accordance with the manufacturer's instructions. Frozen cells were thawed in a water bath at $37^{\circ} \mathrm{C}$ and then seeded in $100-\mathrm{mm}$ culture dishes at a density of $5 \times 10^{3}$ cells $/ \mathrm{cm}^{2}$. The cells were maintained at $37^{\circ} \mathrm{C}$ in humidified $5 \% \mathrm{CO}_{2}$. Twenty-four hours subsequent to seeding, the culture medium was changed to remove the residual DMSO and the unattached cells and was then changed every 2 days. When the cultures reached $90 \%$ confluence, the cells were treated with $0.25 \%$ (w/v) trypsin $/ 0.02 \%$ (w/v) EDTA until all the cells were completely detached. The cells were subsequently resuspended and diluted with ECM, prior to the cell suspension being plated onto 100-mm culture dishes. The culture medium was changed every 2 days. HAECs at $85 \%$ confluence and in the third to sixth passages were used in all the experiments. The cells were seeded in $60-\mathrm{mm}$ culture dishes at a cell density of $5 \times 10^{5}$ cells/dish.

Preparation of cell extracts and western blot analysis. HAECs were pretreated with the specific inhibitor SP600125 $(20 \mu \mathrm{M})$ or SN50 $(18 \mathrm{mM})$ for $1 \mathrm{~h}$ prior to the addition of LPS. Cells were harvested using centrifugation at 2,500 x g for $10 \mathrm{~min}$. The collected cells were subsequently lysed with cell and tissue protein extraction reagent containing protease inhibitor, phosphatase inhibitor and phenylmethanesulfonyl fluoride (PMSF; Kangchen Bio-tech, Shanghai, China). The lysates were then centrifuged at $14,000 \times \mathrm{g}$ for $15 \mathrm{~min}$ at $4^{\circ} \mathrm{C}$ and the debris was removed. The protein concentrations in the lysates were assessed using bicinchoninic acid (BCA) reagents (Beyotime Institute of Biotechnology, Jiangsu, China), in accordance with the manufacturer's protocol.

Protein samples $(40 \mu \mathrm{g})$ were denatured and loaded onto a $10 \%$ sodium dodecyl sulfate-polyacrylamide gel electrophoresis (SDS-PAGE) gel, prior to being electrotransferred onto a $0.2-\mu \mathrm{m}$ polyvinylidene fluoride (PVDF) transfer membrane (Pall Corp., Pensacola, FL, USA). The membranes were subsequently blocked at room temperature with $5 \%(\mathrm{w} / \mathrm{v})$ skimmed milk or $5 \%(\mathrm{w} / \mathrm{v})$ bovine serum albumin (BSA) in Tris-buffered saline and Tween 20 [TBST; Tris- $\mathrm{HCl} 50 \mathrm{mM}$, $\mathrm{NaCl} 150 \mathrm{mM}, 0.05 \%$ (w/v) Tween-20, $\mathrm{pH}$ 7.4] for $\geq 1 \mathrm{~h}$. The blocked membranes were incubated overnight at $4^{\circ} \mathrm{C}$ with the specific antibodies against VCAM-1 (1:100), $\beta$-actin $(1: 5,000)$, p-JNK $(1: 2,000)$, JNK $(1: 1,000), N F-\kappa B(1: 1,000)$ or IкB- $\alpha$ $(1: 1,000)$ in TBST. Subsequent to being washed with TBST, the membranes were incubated with a fluorescent-labeled secondary antibody $(1: 1,000)$ for $1 \mathrm{~h}$, and the immunoreactive products were analyzed using the Odyssey ${ }^{\circledR}$ Infrared Imaging system (LI-COR Biosciences, Lincoln, NE, USA).

Statistical analysis. Statistical analyses were performed using an independent samples t-test with the SPSS 20.0 statistical software package (SPSS, Inc., Chicago, IL, USA). $\mathrm{P}<0.05$ was considered to indicate a statistically significant difference.

\section{Results}

P. gingivalis LPS activated JNK signaling pathway transduction in HAECs. To investigate whether $P$. gingivalis and $E$. coli LPS mediated JNK phosphorylation, the activation of this kinase was examined using an antibody specific for tyrosine-phosphorylated JNK and western blotting. As shown in Fig. $1 \mathrm{~A}$ and $\mathrm{C}$, the $P$. gingivalis LPS-induced phosphorylation of JNK was observed within 15 min subsequent to stimulation and reached a peak at $30 \mathrm{~min}$. In E. coli LPS-treated HAECs, JNK phosphorylation was observed as early as $5 \mathrm{~min}$ and 
A

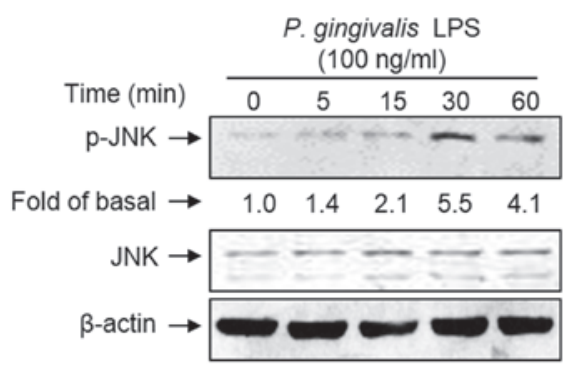

B

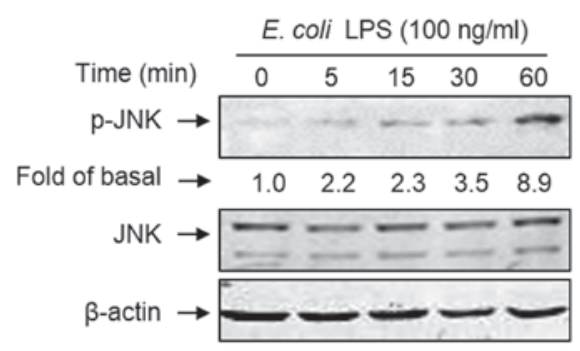

C

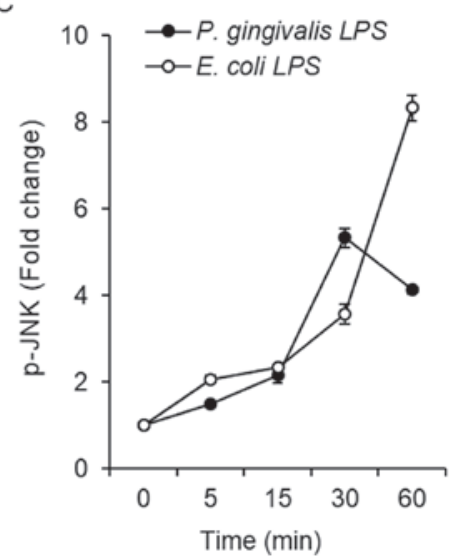

Figure 1. Lipopolysaccharide (LPS) induces the tyrosine phosphorylation of c-Jun N-terminal kinase (JNK) in human aortic endothelial cells (HAECs). Levels of JNK and phosphorylated JNK (p-JNK) were assessed using western blot analysis. HAECs were stimulated with (A) Porphyromonas gingivalis (P. gingivalis) LPS and (B) Escherichia coli (E. coli) LPS for the indicated periods of time. The image shows one of three similar experiments. The intensity of the western blotting bands shown was quantified using scanning densitometry and standardized to the equivalent $\beta$-actin protein levels. (C) Fold induction of p-JNK expression normalized to the equivalent $\beta$-actin protein levels vs. cells cultured in medium alone (basal level). Data are expressed as the mean \pm standard deviation of three independent experiments.

reached the highest level at $60 \mathrm{~min}$, which was the end of the observation period (Fig. 1B and C).

Involvement of JNK in P. gingivalis LPS-induced VCAM-I expression. To demonstrate the involvement of the JNK cell signaling pathway in $P$. gingivalis and $E$. coli LPS-induced VCAM-1 expression in HAECs, SP600125, a selective pharmacological inhibitor (33), was used to block the JNK signaling pathway. As shown in Fig. 2, pretreatment of the HAECs with SP600125 at a concentration of $20 \mu \mathrm{M}$ for $1 \mathrm{~h}$ significantly inhibited the $P$. gingivalis and $E$. coli LPS-induced JNK phosphorylation $(\mathrm{P}<0.01)$. However, SP600125 failed to suppress the upregulation of VCAM-1 induced by the $P$. gingivalis and $E$. coli LPS. Notably, blocking the intracellular JNK signaling pathway with SP600125 significantly elevated the expression of VCAM-1 in LPS-treated HAECs ( $\mathrm{P}<0.01$; Fig. 3).

$P$. gingivalis LPS-activated NF- $\kappa B$ signaling pathway transduction in HAECs. To determine the activation of the transcription factor $\mathrm{NF}-\kappa \mathrm{B}$ in HAECs exposed to $P$. gingivalis and $E$. coli LPS, western blotting was used to assess the degradation of $\mathrm{I} \kappa \mathrm{B}-\alpha$, an indicator of NF- $\kappa \mathrm{B}$ activation, as well as the activation pattern of $\mathrm{p} 65$, a component of $\mathrm{NF}-\kappa \mathrm{B}$, using antibodies specific for $\mathrm{I} \kappa \mathrm{B}-\alpha$ and $\mathrm{NF}-\kappa \mathrm{B}$ p 65 , respectively. As shown in Fig. 4, IкB- $\alpha$ was significantly degraded, corresponding with the upregulation of NF- $\kappa \mathrm{B}$ p 65 , following $30 \mathrm{~min}$ of exposure of the HAECs to $P$. gingivalis and $E$. coli LPS. SN50, a synthetic cell permeable peptide and inhibitor of $\mathrm{NF}-\kappa \mathrm{B}(34)$, has been shown to significantly attenuate the degradation of I $\mathrm{B}-\alpha$ and

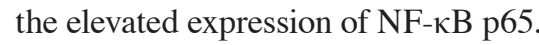

$N F-\kappa B$ inhibitor suppresses $P$. gingivalis LPS-induced VCAM-1 expression. To demonstrate the involvement of the $\mathrm{NF}-\kappa \mathrm{B}$ signaling pathway in $P$. gingivalis and $E$. coli LPS-induced VCAM-1 expression in HAECs, SN50 was used to block the $\mathrm{NF}-\kappa \mathrm{B}$ signaling pathway. As shown in Fig. 5, pretreatment of the HAECs with SN50 at a concentration of $18 \mu \mathrm{M}$ for $1 \mathrm{~h}$
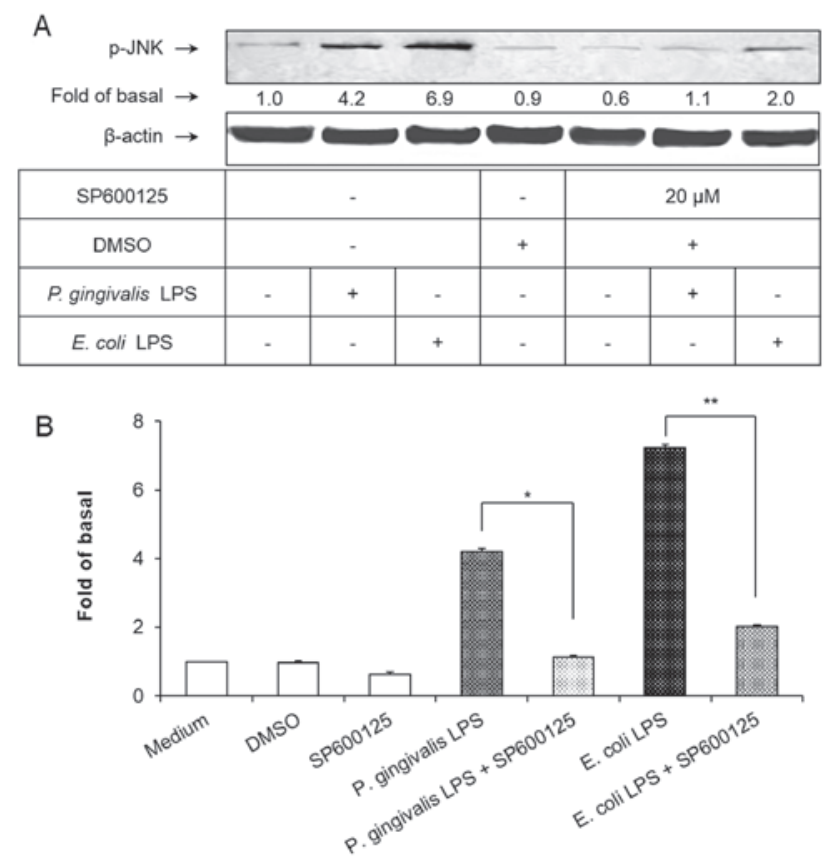

Figure 2. The c-Jun N-terminal kinase (JNK) inhibitor, SP600125, inhibits lipopolysaccharide (LPS)-induced JNK phosphorylation. (A) Cells were preincubated with SP600125 for $1 \mathrm{~h}$ prior to stimulation with Porphyromonas gingivalis (P. gingivalis) LPS (30 min) or Escherichia coli (E. coli) LPS (60 min) at a concentration of $100 \mathrm{ng} / \mathrm{ml}$. The image shows one of three similar experiments. The intensity of the western blotting bands shown was quantified using scanning densitometry and standardized to the equivalent $\beta$-actin protein levels. (B) Fold induction of phosphorylated-JNK (p-JNK) expression compared with the basal level. Data are expressed as the mean \pm standard deviation of three independent experiments. Statistically significant differences were determined using the independent samples t-test. ${ }^{*} \mathrm{P}<0.01,{ }^{* *} \mathrm{P}<0.01$. DMSO, dimethylsulfoxide.

significantly inhibited the induction of VCAM-1 expression in HAECs exposed to $P$. gingivalis and $E$. coli LPS. These results suggested that the activation of $\mathrm{NF}-\kappa \mathrm{B}$ was essential for the $P$. gingivalis and E. coli LPS-induced VCAM-1 expression in HAECs. 

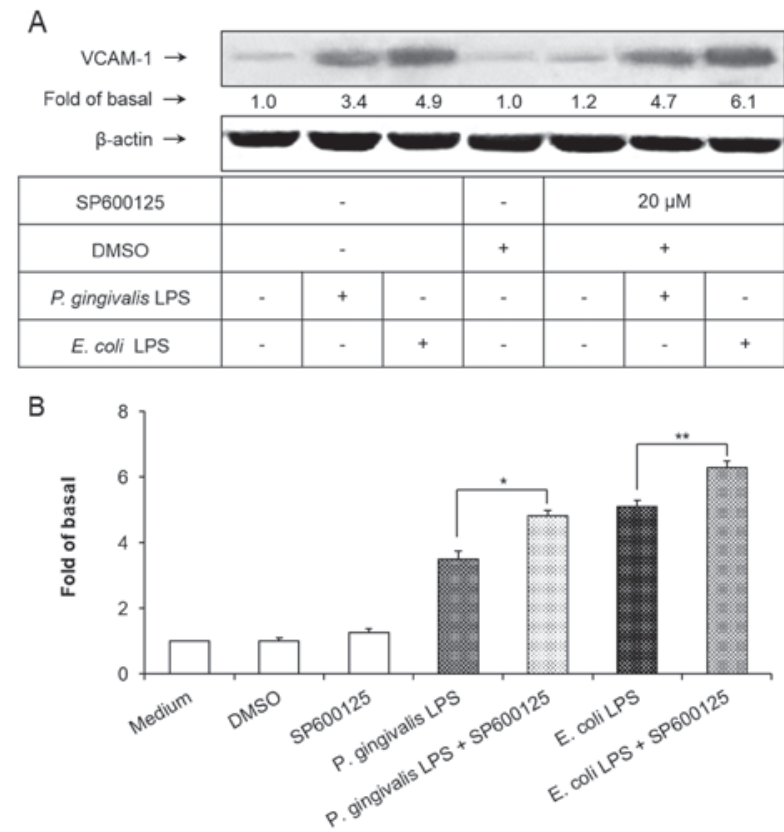

Figure 3. c-Jun N-terminal kinase (JNK) inhibitor, SP600125, inhibits lipopolysaccharide (LPS)-induced vascular cell adhesion molecule-1 (VCAM-1) protein expression. (A) Cells were preincubated with SP600125 for $1 \mathrm{~h}$ prior to stimulation with Porphyromonas gingivalis ( $P$. gingivalis) LPS or Escherichia coli (E. coli) LPS for $16 \mathrm{~h}$. The image shows one of three similar experiments. The intensity of the western blotting bands shown was quantified using scanning densitometry and standardized to the equivalent $\beta$-actin protein levels. (B) Fold induction of VCAM-1 expression compared with the basal level. Data are expressed as the mean \pm standard deviation of three independent experiments. Statistically significant differences were determined using the independent samples t-test. ${ }^{*} \mathrm{P}<0.01,{ }^{* *} \mathrm{P}<0.01$. DMSO, dimethylsulfoxide.

\section{Discussion}

Mononuclear cell (MNC) adhesion to the atherosclerosis-prone vascular endothelium has been suggested to be the initial step of atherosclerosis (15-17). The upregulation of VCAM-1 on the surface of the VECs may be important in the recruitment, rolling, firm adhesion and infiltration of MNCs at sites of inflammation in the vascular endothelium and may accelerate the development of atherosclerosis (35). P. gingivalis LPS has been shown to be an inducer of VCAM-1 $(4,14)$; however, little is known about the intracellular signaling pathways leading to VCAM-1 expression in VECs exposed to $P$. gingivalis LPS. In this study, we investigated the involvement of the JNK and NF- $\mathrm{KB}$ signaling pathways in $P$. gingivalis LPS-induced VCAM-1 expression in HAECs. Using western blotting, we demonstrated that pretreatment of the HAECs with a JNK inhibitor, SP600125, significantly attenuated JNK phosphorylation; however, an upregulation, instead of a downregulation, of $P$. gingivalis LPS-induced VCAM-1 expression was observed in SP600125 pretreated HAECs. Furthermore, the activation of NF- $\mathrm{KB}$ by $P$. gingivalis LPS in the HAECs resulted in VCAM-1 expression, since the activation of NF- $\mathrm{KB}$ and subsequent expression of VCAM-1 was inhibited by an inhibitory peptide of NF- $\kappa B$, SN50. These results suggest that NF- $\kappa B$ was essential for the induction of VCAM-1 protein production in the HAECs exposed to $P$. gingivalis LPS, whereas JNK may be a suppressor of VCAM-1 expression in HAECs.
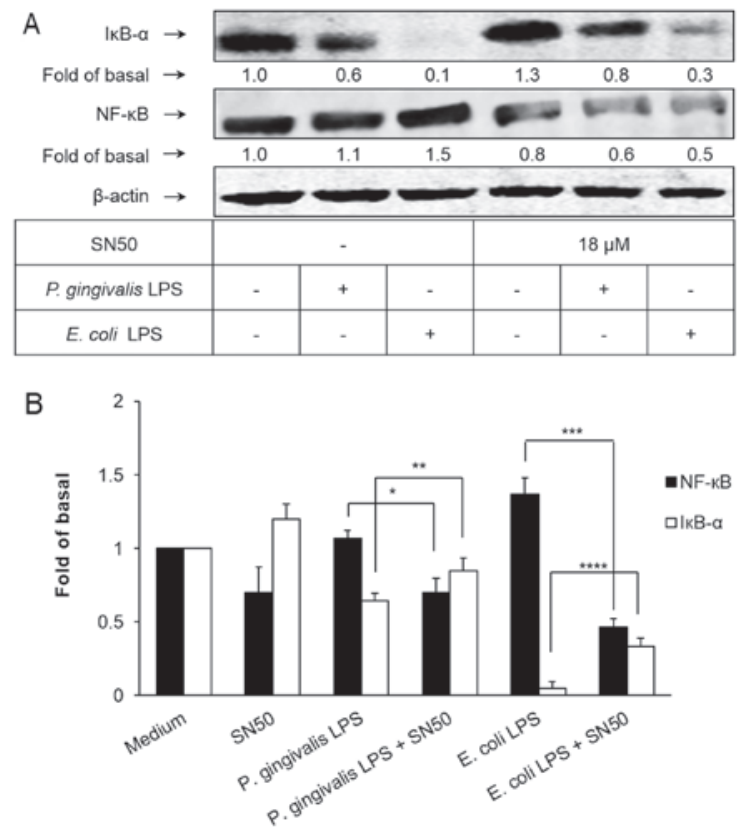

Figure 4. Nuclear factor- $\kappa \mathrm{B}(\mathrm{NF}-\kappa \mathrm{B})$ inhibitor, SN50, inhibits lipopolysaccharide (LPS)-induced degradation of nuclear factor of $\kappa$ light polypeptide gene enhancer in B-cells inhibitor- $\alpha(\mathrm{I} \kappa \mathrm{B}-\alpha)$ and expression of NF- $\kappa \mathrm{B}$ p65. (A) Cells were preincubated with SN50 for $1 \mathrm{~h}$ prior to stimulation with Porphyromonas gingivalis (P. gingivalis) LPS or Escherichia coli (E. coli) LPS for $30 \mathrm{~min}$ at a concentration of $100 \mathrm{ng} / \mathrm{ml}$. The images shows one of three similar experiments. The intensity of the western blotting bands shown was quantified using scanning densitometry and standardized to the equivalent $\beta$-actin protein levels. (B) Fold induction of NF- $\kappa \mathrm{B}$ expression and fold degradation of IкB- $\alpha$ compared with the basal level. Data are expressed as the mean \pm standard deviation of three independent experiments. Statistically significant differences were determined using the independent samples t-test. ${ }^{*} \mathrm{P}<0.01,{ }^{* *} \mathrm{P}<0.01,{ }^{* * *} \mathrm{P}<0.05,{ }^{* * * *} \mathrm{P}<0.01$.
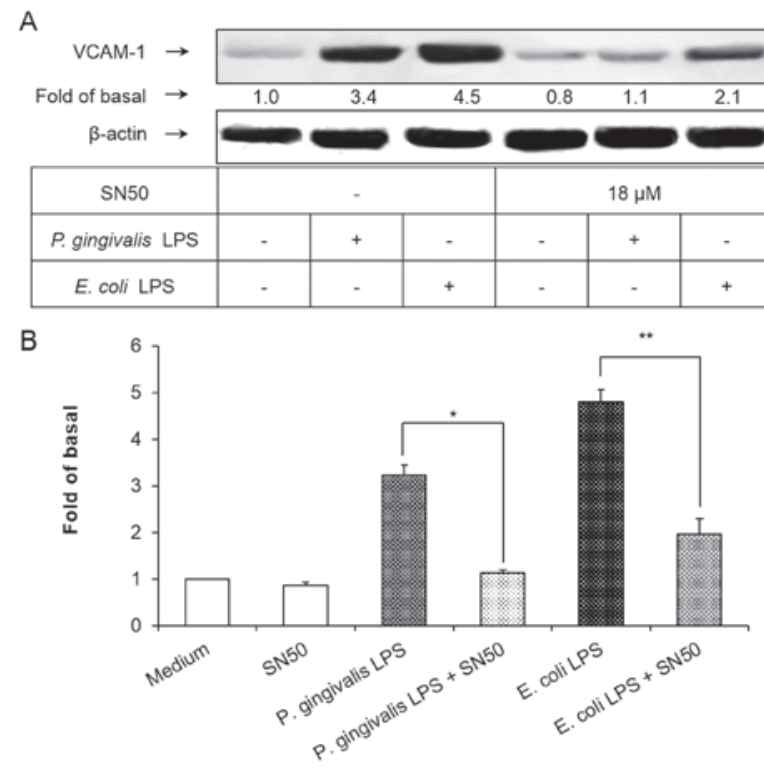

Figure 5. Nuclear factor- $\kappa \mathrm{B}(\mathrm{NF}-\kappa \mathrm{B})$ inhibitor, SN50, inhibits lipopolysaccharide (LPS)-induced vascular cell adhesion molecule-1 (VCAM-1) protein expression. (A) Cells were preincubated with SN50 for $1 \mathrm{~h}$ prior to stimulation with Porphyromonas gingivalis (P. gingivalis) LPS or Escherichia coli (E. coli) LPS for $16 \mathrm{~h}$. The intensity of the western blotting bands shown was quantified using scanning densitometry and standardized to the equivalent $\beta$-actin protein levels. (B) Fold induction of VCAM-1 expression compared with the basal level. Data are expressed as the mean \pm standard deviation of three independent experiments. Statistically significant differences were determined using the independent samples t-test. ${ }^{*} \mathrm{P}<0.01,{ }^{* *} \mathrm{P}<0.01$. 
There are at least three distinct and parallel MAPK pathways, including p38 MAPK, JNK and the extracellular signal-regulated kinase (ERK) pathway (25). It has been demonstrated that the p38 MAPK and JNK pathways mediate signaling stimulated by bacterial components (LPS, lipoteichoic acid), inflammatory cytokines [interleukin-1 $\beta$ (IL-1 $\beta$ ), tumor necrosis factor- $\alpha$ (TNF- $\alpha$ )] and stress factors (osmotic shock, heat shock, $\mathrm{H}_{2} \mathrm{O}_{2}$, UV radiation and DNA-damaging agents), while activation of ERK is important in mediating cell proliferation in response to growth factors and mitogens (36-38). We recently demonstrated that p38 MAPK mediated $P$. gingivalis LPS-induced VCAM-1 expression in HAECs (4). Therefore, the involvement of JNK in this effect was investigated in the present study.

In this study, we demonstrated the activation of the JNK pathway in $P$. gingivalis and E. coli LPS-stimulated HAECs. As shown in Fig. 1, $P$. gingivalis LPS-induced JNK phosphorylation was weaker and reached a peak much earlier than that induced by $E$. coli LPS. Similar results were observed in LPS-induced VCAM-1 expression and NF- $\mathrm{KB}$ activation (Figs. 3-5). Previous studies have revealed discrepancies in the chemical structure of lipid A, the biologically active center of LPS, between $P$. gingivalis and E. coli LPS (32). In addition, it has previously been demonstrated that the endotoxic activities of $P$. gingivalis LPS and its lipid A are relatively weak when compared with those of E. coli-derived LPS and lipid A $(4,13,32)$. The observations in the present study were consistent with the results from those studies, suggesting the potential involvement of $P$. gingivalis LPS in chronic inflammatory processes, such as atherosclerosis and periodontal disease, rather than acute infection, such as sepsis.

Although the present and previous studies have demonstrated the activation of the JNK pathway by $P$. gingivalis and $E$. coli LPS $(26,39)$, the correlation between the activation of JNK and the expression of VCAM-1 has been contradictory in different cell lines. It has been shown that the JNK pathway mediated VCAM-1 expression in human tracheal smooth muscle cells (HTSMCs) exposed to TNF- $\alpha$ (19) and in endothelial cells (EVC304) exposed to E. coli LPS (21). However, Binion et al (20) demonstrated that in human intestinal microvascular endothelial cells (HIMECs) activation of JNK was not a requisite for the induction of VCAM-1 production by E. coli LPS. These results suggest that VCAM-1 is selectively expressed in a cell type and stimulus-specific manner and is mediated through the activation of diverse intracellular signaling pathways. Furthermore, the cell lines used in the majority of the previous studies concerning the activation of JNK in endothelial cells exposed to LPS were venous VECs $(20,21)$. It has been demonstrated that there are genetic variances between artery and vein-derived endothelial cells, which contribute to the different biological and immunological responses to atherosclerotic factors $(13,40,41)$. Of note was the fact that the HUVEC cell line used in a number of studies was the EVC304 cell line $(21,42)$, which has been revealed to be genetically identical to the human bladder cancer-derived epithelial cell line T24/83 and has been suggested to be inappropriate for the study of endothelial cell biology (43). The involvement of the JNK pathway in LPS-induced VCAM-1 expression in HAECs remains to be determined. As shown in Fig. 2, we elucidated that SP600125, an anthrapyrazole and a reversible
ATP-competitive inhibitor of JNK, significantly attenuated the phosphorylation of JNK. However, this was demonstrated to enhance the $P$. gingivalis and $E$. coli LPS-induced protein production of VCAM-1 in HAECs (Fig. 3). These observations suggested that JNK may be an intracellular suppressor for the expression of VCAM-1 in HAECs.

The observation that SP600125 enhanced VCAM-1 expression indicated that the administration of SP600125 with $P$. gingivalis and $E$. coli LPS caused an additive effect on VCAM-1 expression in the HAECs (Fig. 3). This result was consistent with those of previous studies, in which SP600125 was shown to elicit additive effects with TNF- $\alpha$ on VCAM-1 expression in human chondrosarcoma cells and in gingival fibroblasts $(44,45)$, although a study using HK- 2 cells revealed contradictory observations (46). There have been few studies concerning the additive effect of SP600125; therefore, further investigation is required to fully elucidate the underlying molecular mechanism of JNK in VCAM-1 expression.

As extensively studied previously, inflammatory responses following exposure to extracellular stimuli highly depend on the activation of the transcription factor NF- $\mathrm{kB}$ (47-50). The sequestration of NF- $\kappa B$ by I $\kappa$ B- $\alpha$ in the cytoplasm and the phosphorylation of IкB- $\alpha$, leading to the proteasomal degradation of I $\kappa \mathrm{B}-\alpha$, results in the activation and translocation of NF- $\kappa \mathrm{B}$ into the nucleus, a process that is essential in the expression of a number of genes, such as adhesion molecules, in various cell types $(14,48,50)$. In the present study, as shown in Fig. 4, the

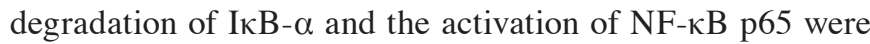
observed following $P$. gingivalis LPS exposure and were also observed to be inhibited by SN50. Furthermore, $P$. gingivalis LPS-induced VCAM-1 expression was almost completely suppressed by SN50 (Fig. 5), indicating that the activation of NF- $\mathrm{kB}$ was essential for the induction of VCAM-1 expression in $P$. gingivalis-stimulated HAECs.

Within the limitations of this study, we have demonstrated that $P$. gingivalis LPS has the ability to accelerate the development of atherosclerosis by upregulating the expression of VCAM-1 in HAECs, a process that is critical in the initiation of atherosclerosis. Furthermore, we demonstrated that $P$. gingivalis LPS was able to activate the JNK and $\mathrm{NF}-\mathrm{\kappa B}$ signaling pathways in HAECs. The activation of the $\mathrm{NF}-\kappa \mathrm{B}$ pathway was essential for the induction of VCAM-1 expression in HAECs exposed to $P$. gingivalis LPS, whereas JNK may be a suppressor of VCAM-1 expression. To the best of our knowledge, this study is the first to investigate the role of JNK in P. gingivalis LPS-induced VCAM-1 expression in HAECs.

\section{Acknowledgements}

This study was supported by grants from the National Natural Science Foundation of China (no. 81100753) and the Science and Technology Commission of Shanghai Municipality (no. 09JC1409100).

\section{References}

1. Lalla E, Lamster IB, Hofmann MA, et al: Oral infection with a periodontal pathogen accelerates early atherosclerosis in apolipoprotein E-null mice. Arterioscler Thromb Vasc Biol 23: 1405-1411, 2003. 
2. Cavrini F, Sambri V, Moter A, et al: Molecular detection of Treponema denticola and Porphyromonas gingivalis in carotid and aortic atheromatous plaques by FISH: report of two cases. J Med Microbiol 54: 93-96, 2005.

3. Zhang MZ, Li CL, Jiang YT, et al: Porphyromonas gingivalis infection accelerates intimal thickening in iliac arteries in a balloon-injured rabbit model. J Periodontol 79: 1192-1199, 2008

4. Liu B, Cheng L, Liu D, et al: Role of p38 mitogen-activated protein kinase pathway in Porphyromonas gingivalis lipopolysaccharide-induced VCAM-1 expression in human aortic endothelial cells. J Periodontol 83: 955-962, 2012.

5. Raetz CR: Biochemistry of endotoxins. Annu Rev Biochem 59: 129-170, 1990.

6. Rice JB, Stoll LL, Li WG, et al: Low-level endotoxin induces potent inflammatory activation of human blood vessels: inhibition by statins. Arterioscler Thromb Vasc Biol 23: 1576-1582, 2003.

7. Stoll LL, Denning GM and Weintraub NL: Potential role of endotoxin as a proinflammatory mediator of atherosclerosis. Arterioscler Thromb Vasc Biol 24: 2227-2236, 2004.

8. Holt SC, Kesavalu L, Walker S and Genco CA: Virulence factors of Porphyromonas gingivalis. Periodontol 2000 20: 168-238, 1999.

9. Geerts SO, Nys M, De MP, et al: Systemic release of endotoxins induced by gentle mastication: association with periodontitis severity. J Periodontol 73: 73-78, 2002.

10. Ide M, Jagdev D, Coward PY, Crook M, Barclay GR and Wilson RF: The short-term effects of treatment of chronic periodontitis on circulating levels of endotoxin, C-reactive protein, tumor necrosis factor-alpha, and interleukin-6. J Periodontol 75: 420-428, 2004

11. Pussinen PJ, Vilkuna-Rautiainen T, Alfthan G, et al: Severe periodontitis enhances macrophage activation via increased serum lipopolysaccharide. Arterioscler Thromb Vasc Biol 24: 2174-2180, 2004

12. Lafaurie GI, Mayorga-Fayad I, Torres MF, et al: Periodontopathic microorganisms in peripheric blood after scaling and root planing. J Clin Periodontol 34: 873-879, 2007.

13. Erridge C, Spickett CM and Webb DJ: Non-enterobacterial endotoxins stimulate human coronary artery but not venous endothelial cell activation via Toll-like receptor 2. Cardiovasc Res 73: 181-189, 2007.

14. Nakamura N, Yoshida M, Umeda M, et al: Extended exposure of lipopolysaccharide fraction from Porphyromonas gingivalis facilitates mononuclear cell adhesion to vascular endothelium via Toll-like receptor-2 dependent mechanism. Atherosclerosis 196 59-67, 2008.

15. Fries JW, Williams AJ, Atkins RC, Newman W, Lipscomb MF and Collins T: Expression of VCAM-1 and E-selectin in an in vivo model of endothelial activation. Am J Pathol 143: 725-737, 1993

16. Cybulsky MI, Iiyama K, Li H, et al: A major role for VCAM-1, but not ICAM-1, in early atherosclerosis. J Clin Invest 107: $1255-1262,2001$

17. Ley K and Huo Y: VCAM-1 is critical in atherosclerosis. J Clin Invest 107: 1209-1210, 2001.

18. Wang CC, Lin WN, Lee CW, et al: Involvement of p42/p44 MAPK, p38 MAPK, JNK, and NF-kappaB in IL-1beta-induced VCAM-1 expression in human tracheal smooth muscle cells. Am J Physiol Lung Cell Mol Physiol 288: L227-L237, 2005.

19. Lee CW, Lin WN, Lin CC, et al: Transcriptional regulation of VCAM-1 expression by tumor necrosis factor-alpha in human tracheal smooth muscle cells: involvement of MAPKs, NF-kappaB, p300, and histone acetylation. J Cell Physiol 207: 174-186, 2006

20. Binion DG, Heidemann J, Li MS, Nelson VM, Otterson MF and Rafiee P: Vascular cell adhesion molecule-1 expression in human intestinal microvascular endothelial cells is regulated by PI 3-kinase/Akt/MAPK/NF-kappaB: inhibitory role of curcumin. Am J Physiol Gastrointest Liver Physiol 297: G259-G268, 2009.

21. Shan Y, Lin N, Yang X, et al: Sulphoraphane inhibited the expressions of intercellular adhesion molecule-1 and vascular cell adhesion molecule-1 through MyD88-dependent toll-like receptor-4 pathway in cultured endothelial cells. Nutr Metab Cardiovasc Dis 22: 215-222, 2012.

22. Han J, Lee JD, Bibbs L and Ulevitch RJ: A MAP kinase targeted by endotoxin and hyperosmolarity in mammalian cells Science 265: 808-811, 1994.

23. Jiang Y, Chen C, Li Z, et al: Characterization of the structure and function of a new mitogen-activated protein kinase (p38beta). J Biol Chem 271: 17920-17926, 1996.
24. Jiang Y, Gram H, Zhao M, et al: Characterization of the structure and function of the fourth member of p38 group mitogen-activated protein kinases, p38delta. J Biol Chem 272: 30122-30128, 1997.

25. Schaeffer HJ and Weber MJ: Mitogen-activated protein kinases: specific messages from ubiquitous messengers. Mol Cell Biol 19: 2435-2444, 1999

26. Lin WN, Luo SF, Lee CW, Wang CC, Wang JS and Yang CM Involvement of MAPKs and NF-kappaB in LPS-induced VCAM-1 expression in human tracheal smooth muscle cells. Cell Signal 19: 1258-1267, 2007.

27. Watanabe K, Yilmaz O, Nakhjiri SF, Belton CM and Lamont RJ: Association of mitogen-activated protein kinase pathways with gingival epithelial cell responses to Porphyromonas gingivalis infection. Infect Immun 69: 6731-6737, 2001.

28. Choi EK, Park SA, Oh WM, et al: Mechanisms of Porphyromonas gingivalis-induced monocyte chemoattractant protein-1 expression in endothelial cells. FEMS Immunol Med Microbiol 44: 51-58, 2005.

29. Lee SD, Wu CC, Kuo WW, et al: Porphyromonas gingivalisrelated cardiac cell apoptosis was majorly co-activated by p38 and extracellular signal-regulated kinase pathways. J Periodontal Res 41: 39-46, 2006.

30. Lazaar AL, Albelda SM, Pilewski JM, Brennan B, Pure E and Panettieri RA Jr: T lymphocytes adhere to airway smooth muscle cells via integrins and CD44 and induce smooth muscle cell DNA synthesis. J Exp Med 180: 807-816, 1994.

31. Hamada S, Koga T, Nishihara T, Fujiwara T and Okahashi N: Characterization and immunobiologic activities of lipopolysaccharides from periodontal bacteria. Adv Dent Res 2: 284-291, 1988.

32. Ogawa $\mathrm{T}$ and Yagi T: Bioactive mechanism of Porphyromonas gingivalis lipid A. Periodontol 2000 54: 71-77, 2010.

33. Bennett BL, Sasaki DT, Murray BW, et al: SP600125, an anthrapyrazolone inhibitor of Jun N-terminal kinase. Proc Natl Acad Sci USA 98: 13681-13686, 2001.

34. Lin YZ, Yao SY, Veach RA, Torgerson TR and Hawiger J: Inhibition of nuclear translocation of transcription factor NF-kappa B by a synthetic peptide containing a cell membrane-permeable motif and nuclear localization sequence. J Biol Chem 270: 14255-14258, 1995.

35. Muller WA: Mechanisms of leukocyte transendothelial migration. Annu Rev Pathol 6: 323-344, 2011

36. Eder J: Tumour necrosis factor alpha and interleukin 1 signalling: do MAPKK kinases connect it all? Trends Pharmacol Sci 18 319-322, 1997.

37. Robinson MJ and Cobb MH: Mitogen-activated protein kinase pathways. Curr Opin Cell Biol 9: 180-186, 1997.

38. Yang CM, Luo SF, Wang CC, et al: Tumour necrosis factor-alphaand interleukin-1beta-stimulated cell proliferation through activation of mitogen-activated protein kinase in canine tracheal smooth muscle cells. Br J Pharmacol 130: 891-899, 2000.

39. Diya Z, Lili C, Shenglai L, Zhiyuan G and Jie Y: Lipopolysaccharide (LPS) of Porphyromonas gingivalis induces IL-1beta, TNF-alpha and IL- 6 production by THP-1 cells in a way different from that of Escherichia coli LPS. Innate Immun 14 99-107, 2008 .

40. Swift MR and Weinstein BM: Arterial-venous specification during development. Circ Res 104: 576-588, 2009.

41. Wu X, Zou Y, Liang Y, et al: COUP-TFII switches responses of venous endothelium to atherosclerotic factors through controlling the profile of various inherent genes expression. J Cell Biochem 112: 256-264, 2011.

42. Zhang D, Zheng H, Zhao J, et al: Porphorymonas gingivalis induces intracellular adhesion molecule-1 expression in endothelial cells through the nuclear factor-kappaB pathway, but not through the p38 MAPK pathway. J Periodontal Res 46: 31-38, 2011

43. Brown J, Reading SJ, Jones S, et al: Critical evaluation of ECV304 as a human endothelial cell model defined by genetic analysis and functional responses: a comparison with the human bladder cancer derived epithelial cell line T24/83. Lab Invest 80: 37-45, 2000.

44. Ju JW, Kim SJ, Jun CD and Chun JS: p38 kinase and c-Jun $\mathrm{N}$-terminal kinase oppositely regulates tumor necrosis factor alpha-induced vascular cell adhesion molecule-1 expression and cell adhesion in chondrosarcoma cells. IUBMB Life 54: 293-299, 2002.

45. Hosokawa Y, Hosokawa I, Ozaki K, Nakae H and Matsuo T: Cytokines differentially regulate ICAM-1 and VCAM-1 expression on human gingival fibroblasts. Clin Exp Immunol 144: 494-502, 2006 
46. Ho AW, Wong CK and Lam CW: Tumor necrosis factor-alpha up-regulates the expression of CCL2 and adhesion molecules of human proximal tubular epithelial cells through MAPK signaling pathways. Immunobiology 213: 533-544, 2008.

47. Newton R, Kuitert LM, Bergmann M, Adcock IM and Barnes PJ: Evidence for involvement of NF-kappaB in the transcriptional control of COX-2 gene expression by IL-1beta. Biochem Biophys Res Commun 237: 28-32, 1997.

48. Chen CC, Chen JJ and Chou CY: Protein kinase calpha but not p44/42 mitogen-activated protein kinase, p38, or c-Jun $\mathrm{NH}(2)$-terminal kinase is required for intercellular adhesion molecule-1 expression mediated by interleukin-1beta: involvement of sequential activation of tyrosine kinase, nuclear factor-kappaB-inducing kinase, and IkappaB kinase 2. Mol Pharmacol 58: 1479-1489, 2000.
49. Guha M and Mackman N: LPS induction of gene expression in human monocytes. Cell Signal 13: 85-94, 2001.

50. Bian ZM, Elner SG, Yoshida A, Kunkel SL, Su J and Elner VM: Activation of $\mathrm{p} 38$, ERK1/2 and NIK pathways is required for IL-1beta and TNF-alpha-induced chemokine expression in human retinal pigment epithelial cells. Exp Eye Res 73: 111-121, 2001. 